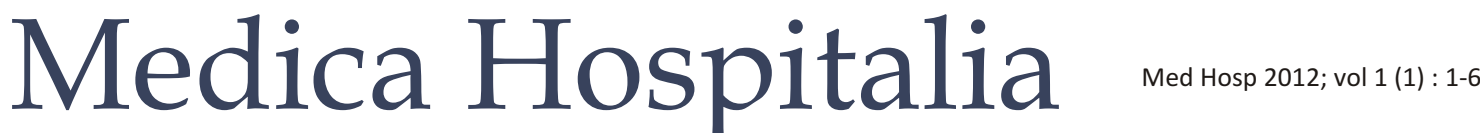

Review Article

Medical Progress

\section{Epilepsy surgery in Indonesia: Achieving better result with limited resources}

\author{
Zainal Muttaqin \\ Department of Neurosurgery Faculty of Medicine Diponegoro University/Dr. Kariadi Hospital Semarang, Indonesia
}

\begin{abstract}
Background: Even with modern medication, 30 to $40 \%$ of epilepsy patients will be intractable and this condition leads to cognitive and psychosocial decline, resulting in worse quality of life and higher mortality. With $0.5-0.6 \%$ prevalence, there will be about 1.5 million epileptic in Indonesia, about 440.000 will be intractable, and 220.000 of them are potential candidates for epilepsy surgery (ES). A decade has passed since the first ES performed on July 1999, and the number increases every year reaching 35-47 ES per year in 2007-2009. Despite the excellent result shown, all of these ES were still performed in Semarang (Diponegoro University) while the patients were from all part of Indonesia. The major reason behind the unavailability of ES in most part of the country should be discussed for the sake of future development of ES in Indonesia.

Material: Epilepsy surgery was started in July 1999 with anterior temporal lobectomy for a 34 Y-old female with left mesial temporal sclerosis (MTS) causing a long standing intractable seizures. The number of cases increases every year. Until the end of 2009, there were 238 cases of epilepsy surgery, including 212 anterior temporal lobectomies. Among these, 106 cases had been follow up more than 36 months, and evaluated for surgical results.

Methods: To evaluate the patient's selection and the presurgical evaluation, we divide the ES cases into the first 5 years ( 56 cases) and the recent 5 years (182 cases). But for the purpose of evaluating surgical results, only those with at least 36 months postoperative follow-up were included (106 cases) and grouped into those operated before or after the age of 25 Y-old (group A and group B), and into those operated before or after the length of epilepsy of 10 years (group I and group II).

Results: For the first five years-period, decision to operate were based on MRI and routine interictal EEG in 54 out of 56 TLE cases. One patient had long-term ictal EEG and another had subdural grid EEG implanted, since MRI in both patients showed visually normal MRI. For the last five years, decision to operate were based on MRI and routine EEG in 91 out of 156 TLE cases. Long term ictal EEG were performed in 46 patients, subdural grid EEG in 10 patients, PET study in 7 patients, and EcoG in 2 patients. The overall seizure free (SF) rate were $70.75 \%$, but if grouped according to patient's age at surgery (less than or over $25 \mathrm{Y}$-old), the SF rates were $75.4 \%$ vs $66.04 \%$ respectively. So did if grouped according to length of disease (less than or more than 10 years), the SF rates were $78.72 \%$ vs $64.40 \%$ respectively.

Conclusion: MRI plays very important role to decide the side of the epileptic temporal side, but this role is decreasing as it was $96.4 \%$ during the beginning five years to become $58.34 \%$ for the last five years. This means that we are working on more difficult epilepsy cases recently. SF rate was significantly higher for those who was operated at younger age and for those with shorter duration of epilepsy. This means that surgery should be offered earlier for those intractable TLE patients with obvious focus on MRI.
\end{abstract}

\section{INTRODUCTION}

Despite the availability of modern antiepileptic drugs (AEDs), up to $30-40 \%$ of epilepsy patients continue to have seizures ${ }^{1,2}$ and approximately half of these are potential candidates for surgery. ${ }^{3}$ Complex partial seizures with seizure focus mostly in the temporal lobe made up most of these intractable cases. ${ }^{4}$ Surgery to abolish seizures or epilepsy surgery (ES) is recommended for mesial temporal lobe epilepsy (MTLE), which is possibly the most common form of human epilepsy and the most refractory to AEDs. ${ }^{5.7}$ Approximately 65\% patients are seizure free (SF) after surgery, while another $21 \%$ improved. ${ }^{8,9}$ The first randomized controlled trials on patients with refractory epilepsy ${ }^{10}$ has shown that in temporal lobe epilepsy (TLE), surgery is superior to optimized AED therapy, with more than $64 \%$ SF with surgery and $8 \%$ SF with medical therapy.

In TLE, the surgical procedure is a standardized anterior temporal lobectomy (ATL) including amygdalohippocampectomy. Magnetic resonance imaging (MRI) is currently the best imaging modality for patient with partial epilepsy. ${ }^{11-14}$ Visual MRI has the capability to identify mesial temporal sclerosis (MTS) in $80-90 \%$ of patients, and nearly $90 \%$ patients will be SF after ATL. ${ }^{15-19}$ Quantitative MRI studies, showing unilateral volume loss, further improves the sensitivity of detecting epileptogenic temporal lobe..$^{20-21}$ The volumetric MRI is 
particularly useful in patients with bilateral hippocampal atrophy, subtle unilateral atrophy, and symmetrically appearing hippocampi..$^{21-22}$

Assuming an epilepsy prevalence rate similar to elsewhere at $0.5-0.6 \%$, there are about 1.5 million people with epilepsy (PWE) in the 230 million Indonesia's populations. Among them, about 440,000 are likely to be refractory to AEDs and half of them, about 220,000 may benefit from ES. Surgery to abolish seizures had been performed at Diponegoro University (Dr. Kariadi) and Telogorejo Hospitals in Semarang for a decade. Until December 2009, there were 238 cases of ES, including 212 temporal lobectomies. Surgical indications and presurgical evaluations were compared first half period (1999-2004) and the second half (2005-2009). For the purpose of evaluating the results of ES, only those with follow-up at least 36 months were included. There were 106 TLE cases grouped according to their age at surgery and length of epilepsy before surgery.

Despite the excellent results shown by ES elsewhere, including those reported from Semarang, ${ }^{23}$ there is still underutilization of ES as an effective method to abolish seizures, especially in developing countries. Wieser and Silfvenius 24 revealed that in 1999 only 26 out of 142 developing countries had at least one center performing ES, despite the fact that developing countries are home of around $90 \%$ of the PWE worldwide. India, with a population of over 1 billion, has had around 1200 cases of ES, performed at two major centers. $^{25}$ Malaysia, with 25.3 million population estimates about 3000 surgical candidates and only 105 ES had been performed at Hospital UKM, Kuala Lumpur. ${ }^{26}$ The situations in Indonesia is more or less mirrors the scenario in these developing countries. The major reasons behind the unavailability of ES in most part of the country, and the future direction to overcome this 'surgical gap' will be discussed.

\section{MATERIALS AND METHODS}

Until the end of 2009, there were 238 cases of ES, ages 3-48 year-old, consisted of 136 males and 102 females.

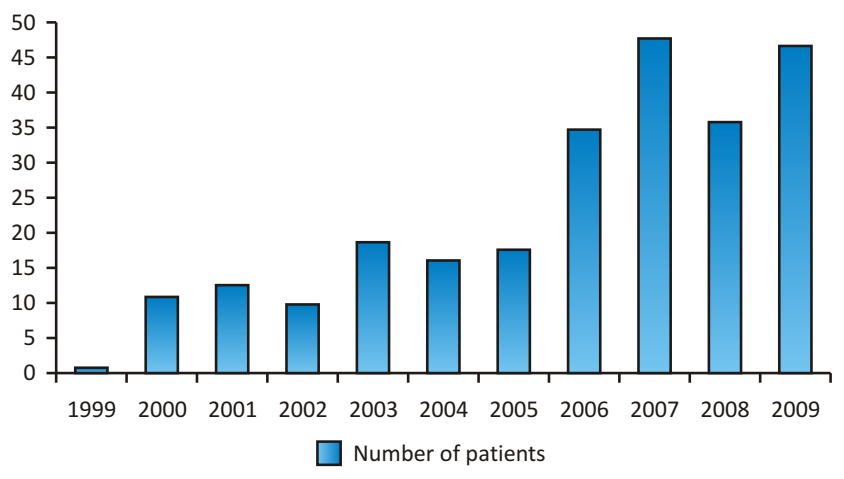

Figure 1. The number of operated epilepsy cases is increasing every year, reaching 35-47 cases per year during the last three years
Among these, there were 212 TLE cases, and 106 of them had post-operative follow-up more than 36 months, and evaluated for surgical results.

Data showed that the number of ES is growing quickly from about 10 patients a year in the first five years to become around 40 patients a year in the recent five years (Figure 1). From the patient's educational level, it is found that very high proportion (53\%) of them who finished high school or even college, while only $28 \%$ were really left behind or under educated (Figure 2).

All patients underwent a standardized ATL as mentioned elsewhere (Figure 3). So that all kind of presurgical evaluations were performed to decide the epileptic side of the temporal lobe. Patients were divided into the first five years, from 1999 to 2004 (56 cases) and the recent five years, from 2004 to 2009 (156 cases), and both groups were compared in types of pre-surgical evaluation, especially regarding the role of MRI in deciding which side of the temporal lobe to be operated.

The seizure elimination rate were evaluated based on Engel's outcome scale as follows: Class I: seizure free or free of disabling seizures; Class II: not more than two attacks per year; Class III : Seizure frequency decreases at least more than $75 \%$, and Class IV: Seizure frequency decreases less than $75 \%{ }^{27} \mathrm{ES}$ were regarded as beneficial for those with Engel's Class I-III. ${ }^{4,28}$ For the purpose of evaluating surgical results, only those with at least 36 months postoperative follow-up were included (106 cases). In order to elucidate the effect of age at surgery, and the length of epilepsy before surgery, they were grouped into those operated before the age of 25 (Group A) and those operated after the age of 25 (Group B), and into those operated when the length of epilepsy were less than 10 years (Group I), or after the length of epilepsy is more than10 years (Group II). Simple statistical methods (student $t$ test) were applied to compare between Group A and Group B and between Group I and Group II.

\section{RESULTS}

There were 56 TLE cases operated during the first five years period (1999-2004), and 54 of them (96.4\%)

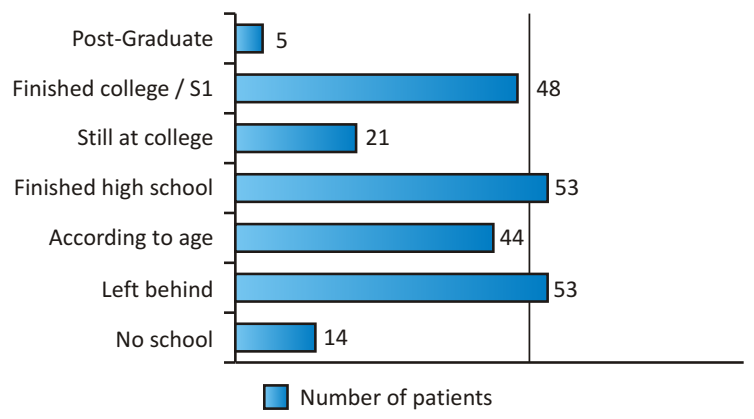

Figure 2. People with Epilepsy (PWE) does not always 'left behind' or under educated, in fact 55\% finished at least High School or Higher education, and only $27 \%$ does under-educated 


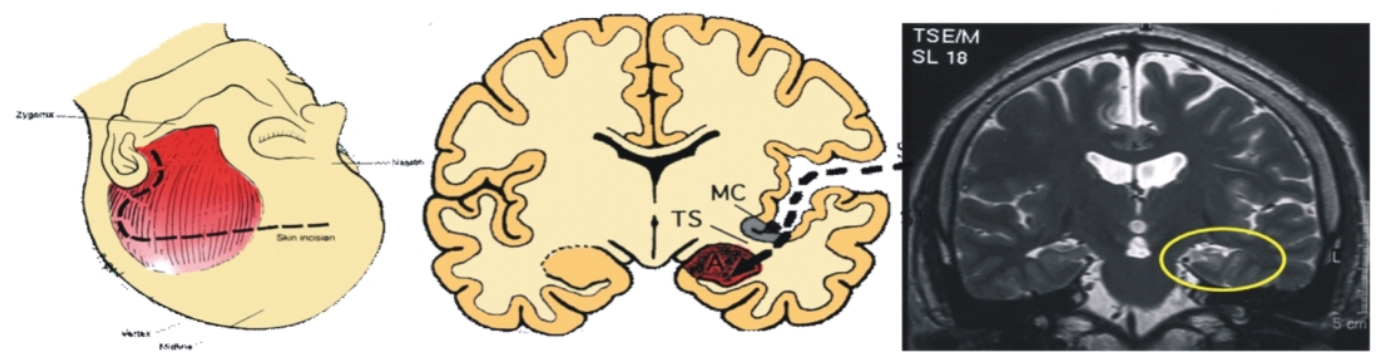

Figure 3. Surgery for TLE is a standardized Anterior Temporal Lobectomy, so that the purpose of pre-surgical evaluation is to decide the epileptic side of the temporal lobe, and MRI plays a very important role

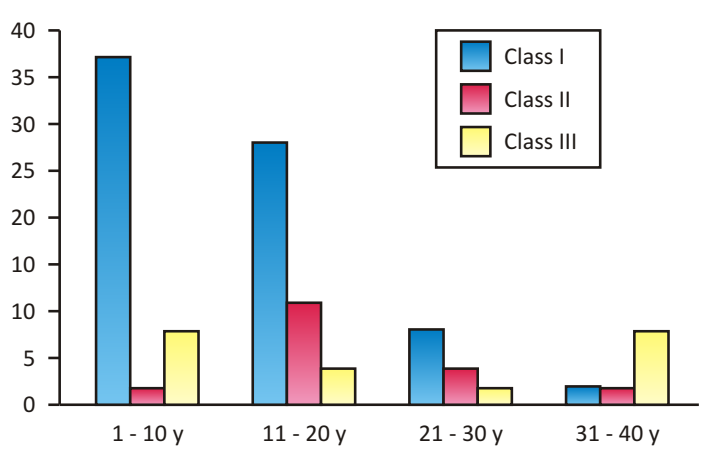

Figure 4. The overall outcome of 106 cases with follow up at least 24 months. In relation to length of epilepsy before surgery. Although the Engel's Class I (free from disabling seizures) reaches $70.75 \%$ but there's a trend that the number of Class II and Class III results increases as the length of epilepsy is longer

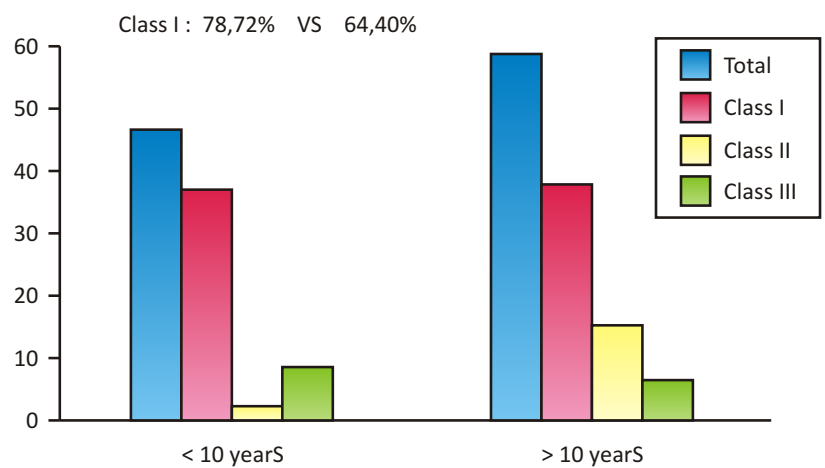

Figure 6. Comparison between Group I ( operated when length of epilepsy is $<10$ years) and Group II (operated when length of epilepsy is $>10$ years). There's also a statistically significant difference in seizure free rate of 78.72\% for Group I versus $64.40 \%$ for Group II

were operated based on semiology of TLE, routine interictal electroencephalography (EEG), and anatomic MRI evaluation. Their MRI showed MTS in 50 cases, Temporal lobe glioneuronal tumor in 3 cases, and hemispheric hemiatrophy in one case. The other two cases had normal MRI, the so called MRI negative cases, and decision on the epileptic side of the temporal lobe were based on long term EEG in one case, and invasive subdural grid EEG in the other case.

ES were performed on 156 TLE cases during the recent five years period (2004-2009). Besides epilepsy

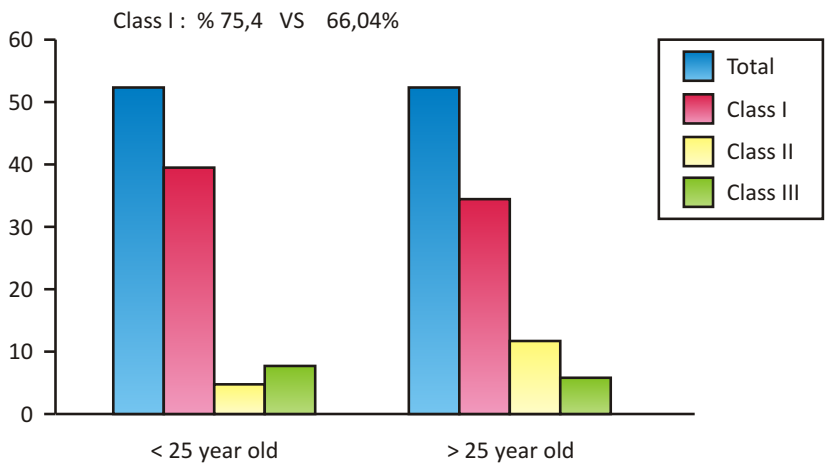

Figure 5. Comparison between Group A (operated at age $<25$ year-old), and Group B (operated at age > 25 year-old). There's a statistically significant difference in seizure free rate of $75.04 \%$ for Group A versus $66.04 \%$ for Group B.

semiology, anatomic MRI plays a role in the decision of the epileptic temporal side in only 91 cases of them (58.34\%). It means that the other 65 cases showed doubtful MRI laterality, or visually normal MRI. These cases were operated based on long term ictal EEG in 46 cases, invasive subdural grid EEG in 10 cases, direct cortical monitoring during surgery or electrocorticography (ECoG) in 2 cases, and 28-Fluorodeoxy Glucose Positron Emission Tomography (FDG-PET) study in the 7 cases.

For those with follow up period of at least 36 months (106 cases), the overall SF rate was $70.75 \%$ (Figure 4). Comparison between Group A and Group B showed that the number of Engel's Class I (free of disabling seizures) were significantly higher $(75.4 \%$ versus $66.4 \%)$ for those operated at the age of less than 25 year-old (Fig. 5). Similar result were seen when comparing Group I and Group II, which showed significantly higher number of SF rate $(78.72 \%$ versus $64.40 \%)$ for those operated when the length of epilepsy was less than 10 years (Figure 6).

Surgical complications were epidural empyema in 5 cases, which needed surgical debridement to remove infected bone flaps, temporary hemiparesis in one case related to coagulation of branch of choroidal artery which recovered after three months of rehabilitation, and depression in three cases which needed psychiatric consultation and one even had a suicide attempt. 


\section{DISCUSSION}

Surgery to abolish seizures had never been performed before the year of 1999, so that we had to start with good and typical MTLE cases which will show good surgical results. Patients with typical MTLE semiology whose MRI showed unilateral hippocampal sclerosis/MTS were selected. We performed 56 ES during the first five years, and $96.4 \%$ were based simply on seizure semiology and the presence of unilateral epileptogenic lesion on MRI. Zentner et $\mathrm{al}^{4}$ has shown that MRI's sensitivity reaches $98.7 \%$ for neoplastic lesion, $76.6 \%$ for non-neoplastic lesions such as cavernous angioma, and $69.2 \%$ for MTS/ hippocampal sclerosis. For the recent five years, there were only $58.34 \%$ of our ES cases which were based simply on seizure semiology and MRI. Decreasing role of MRI for deciding the epileptic side of the temporal lobe means that we are currently working on more difficult epilepsy cases, which were left behind during our first five years. These difficult cases with doubtful MRI laterality needed other modalities to decide exactly the side of the epileptic focus, and long-term ictal EEG and invasive subdural grid EEG would be the modalities of choice, performed in 56 out of 65 or $86.15 \%$ of difficult cases at our centers.

Routine interictal EEG with 30 minutes recording didn't do much help since $60 \%$ showed bilateral interictal discharges despite good outcome after unilateral temporal lobectomy. ${ }^{29-32}$ Lateralized interictal discharges is seen in only $41 \%$ patients. ${ }^{32}$ Interictal discharges may also incorrectly letaralize the temporal foci in $10-20 \%$ patients ${ }^{18,19,33}$. The discharges from medial temporal lobe may have been propagated to the lateral cortex of the opposite sides resulting in incorrect localization. ${ }^{34,35}$ Using long-term ictal EEG with video monitoring, accurate localization of the epileptic foci is possible in $65-70 \%$ of TLE patients. ${ }^{34,35}$

Engel's Outcome Scale ${ }^{27}$ is the most widely used reference for the results of ES. According to Engel's criteria, all of our patients were Class I to III, means that all benefited from the surgery. Those with Engel's Class I gained most from ES, which in our cases reached 70.75\% for those with follow up at least 36 months. This result is consistent with others, where $65-75 \%$, patients achieve seizure freedom after anterior temporal lobectomy with amygdalo-hippocampectomy and another $20 \%$ has meaningful improvement. . $^{27,36-40}$ Wyler AR 28 reported that the probability of SF reached $90 \%$ after one year, and increased to $94 \%$ after two years. So that evaluation at the end of $2^{\text {nd }}$ post-operative year is a good predictor of the long-term seizure outcome.

Our data regarding patient's level of education had eliminated one social stigma pretending that people with epilepsy (PWE) are usually 'left behind' or under educated. More than half of our 238 operated cases finished high school or even university, while the under educated ones only $28 \%$. For PWE with good level of education hoping to start a professional career will be much disturbed by even a very rare seizure, occurring once is several years.

Our studies has shown that ES can achieve good results in developing countries with limited resources, as also reported by Chinvarum ${ }^{41}$ and Srikijvilaikul ${ }^{42}$ from Thailand, and Panda ${ }^{43}$ from South India. Delay in surgery results in psychosocial, educational, and vocational problems which are difficult to overcome even though the seizures have been eliminated by surgery. ${ }^{44,45}$ Our results also showed that the SF rate is significantly higher in those operated at a younger age, and those whose length of disease is shorter. This means that surgery is not the last treatment modality for epilepsy, after everything else had failed. So that simple and non-invasive pre-surgical evaluation such as MRI with specific protocol should be justified for PWE showing typical temporal lobe semiology or other localization-related semiology, and ES should be offered earlier for TLE or other partial/ localization-related epilepsy with obvious focus on MRI. Because of these reasons, there should be early identification of medically refractory epilepsy and prompt referral for surgical treatment. ${ }^{46}$ Based on economic cost, it has been shown that in a developing country like India or Indonesia, one-time successful surgery is almost five times less expensive than a lifetime of unsuccessful medical treatment. ${ }^{47}$

Recent studies had elucidated several biological basis for refractoriness, such as the presence of structural abnormalities, particularly MTS/hippocampal sclerosis and cortical dysplasia. ${ }^{48-50}$ Therefore, besides its role for detecting the epileptic side of the temporal lobe, MRI has an important role in identifying those partial epilepsy cases that will finally become intractable. Even with 0.5 Tesla MRI, the rate of detection of temporal lobe pathology related to epilepsy reached $79 \%$, while MTS alone was detected in $59 \%$ cases. $^{51}$

\section{Future Direction of Epilepsy Surgery in Indonesia}

Despite the fact that developing countries are home of around $90 \%$ of people with epilepsy in the world ${ }^{24}$ only 26 of 142 developing countries had at least one epilepsy surgery center. As shown from the situations in India, ${ }^{25}$ with only two major centers performing ES, and in Malaysia, ${ }^{26}$ with only one center, there is still wide 'surgical gap' between the number of intractable epilepsy cases based on epidemiological data and the number of actual ES performed every year. Absence of epilepsy surgery facilities in most part of the country, such as in Indonesia, is one of the major reasons behind the lack of awareness among referring physicians and even neurologists about the benefits of ES. In situations that they are far from understanding about ES and almost never been exposed or having contact with any ES case, their fears about surgery are exaggerated. This lack of 
awareness thereby leads to non-referrals or delayed referrals. Data from our 238 surgical cases shows the mean duration of suffering from intractable epilepsy before being operated was around 15 years. But, these patients who were operated even after 15 years were the more fortunate ones, compared to tens of thousands who never heard about ES. The majority of PWE who were finally being operated at our center were self-referred and they knew about ES from the mass media, including internet, or hearing other patient's 'surgical experiences'. On the other hand, all the new and expensive antiepileptic drugs (AEDs) are extensively marketed, in spite of the fact that the chance of new AEDs, including levetiracetam, will control these intractable patients is at best around $16 \%,{ }^{52}$ as compared to $70-90 \%$ with ES. ${ }^{15-19}$

Therefore, to popularize epilepsy surgery, the protocols should be simplified so that it might be implemented in centers with limited resources. The largest portion of epilepsy surgery burden in developing countries such as Indonesia is TLE, predominantly related to MTS. Of the $238 \mathrm{ES}$ performed in Semarang for the past ten year, $212(89.1 \%)$ were TLE underwent anterior temporal lobectomies. As shown by our experience during the first five years (1999-2004), almost all intractable epilepsy cases were operated based simply on seizure semiology and MRI study. There is no difficult pre-surgical evaluation needed at all. The presence of unilateral temporal lobe lesion with concordant seizure semiology is a good indication for ES with good results. ${ }^{23}$

The hardware for performing an ES would be any neurosurgical center with microneurosurgical facilities, staffed with well trained epilepsy surgeon and epileptologist. These 'primary' epilepsy surgery centers, capable of performing surgery for simple TLE cases, should be present at least one in each province in Java (each has more than 30 million population), and one for any large islands in the archipelago. It will be perfect if these centers were also equipped with good MRI and EEG for routine examinations. Of course that Semarang (Diponegoro University Hospital) should be one larger 'secondary' epilepsy center capable of performing surgery and pre-surgical evaluations for more difficult epilepsy cases such as shown by our experiences during the recent five years (2004-2009). This referral ES center should be equipped with a high resolution MRI, longterm Video EEG, and facilities for invasive EEG monitoring, and supported by complete epilepsy team consisted of neurologist, child neurologist, psychiatrist, and psychologist, and rehabilitation specialist.

Therefore, in regards to surgery for TLE, it is imperative that hospitals planning to be a 'primary' epilepsy surgery centers ensure that their appointed neurosurgeons are adequately trained by joining as many ES related activities as possible in Semarang. The two centers actively performing ES in India train a minimum of 10 neurosurgeons every year to serve in various parts of the country. Even if only half of them could finally initiate ES, it would result in significant growth of Epilepsy Surgery services across the country. The future would be that epilepsy surgery will be part of a comprehensive management of epilepsy in all part of Indonesia, and at least every neurosurgery service in the setting of a teaching hospital should be capable of performing surgery for simple epilepsy cases such as TLE caused by MTS/ hippocampal sclerosis. The question "is this patient intractable?" will someday be changed into "does the patient has a surgically amenable epileptogenic lesion in the brain?", since delayed referral to ES centers means 'lost of years'. ${ }^{53,54}$

\section{CONCLUSION}

MRI plays very important role to decide the side of the epileptic temporal side, but this role is decreasing as it was $96.4 \%$ during the beginning five years to become $58.34 \%$ for the last five years. This means that we are working on more difficult epilepsy cases recently. SF rate was significantly higher for those who was operated at younger age and for those with shorter duration of epilepsy. This means that surgery should be offered earlier for those intractable TLE patients with obvious focus on MRI.

\section{REFERENCES}

1. Cockerell OC, Johnson AL, Sander JWAS, Hart YM, and Shorvon SD. Remission of Epilepsy: results from the national general practice study of epilepsy. Lancet 1995; 346: 140-4.

2. Kwan P, Brodie MJ. Early identification of refractory epilepsy. N Eng J Med 2000; 342:314-9.

3. Engel J Jr, Shewmon DA. Overview: who should beconsidered a surgical candidate? In: Engel J Jr, ed: Surgical treatment of the epilepsies. 2nd Ed. New York: Raven Press, 1993: 23-34.

4. Zentner J, Hufnagel A, Wolf HK, et al. Surgical treatment of temporal lobe epilepsy; clinical, radiological, and histopathological findings in 178 patients. J Neurol Neurosurg Psychiatry 1995; 58: 666-73.

5. Engel J Jr. Current concept: surgery for seizures. N Eng J Med 1996;334:647-52.

6. Engel J. Jr. Etiology as a risk factor for medically refractory epilepsy: a case for early surgical intervention. Neurology 1998; 51:1243-4.

7. Schmidt D, Bertram E, Ryvlin P, Luders HO. The impact of temporal lobe surgery on cure and mortality of drug-resistant epilepsy: summary of a workshop. Epilepsy Res 2003; 56: 83-4.

8. Engel J.Jr, Wiebe S, French J, et al. Practice parameter. Temporal lobe and localized neocortical resections for epilepsy. Report of the Quality Standards Subcommittee with the American Academy of Neurology in Association with the American Epilepsy Society and the American Association of Neurological Surgeons. Neurology 2003; 60:538-47.

9. McIntosh AM, Wilson SJ, Berkovic SF. Seizure outcome after temporal lobectomy: current research practice and findings. Epilepsia 2001; 42: 1288-307.

10. Wiebe S, Blume WT, Girvin JP, Eliasziw M. A randomized controlled trial of surgery for temporal lobe epilepsy. N Eng J Med 2001;345:311-8. 
11. Dowd CF, Dillon WP, Barbaro NM, et al. Magnetic resonance of intractable complex partial seizures: pathologic and electroencephalographic correlation. Epilepsia 1991; 32: 454459

12. Brooks BS, King DW, Gammal TE, et al. Magnetic resonance imaging in patients with intractable complex partial epileptic seizures. AJNR Am J Neuroradiol 1990; 11: 93-9.

13. Jabbari B, Gunderson $\mathrm{CH}$, Wippold F, et al. Magnetic resonance imaging in partial complex epilepsy. Arch Neurol 1986; 43: 86972.

14. Tien RD, Felsberg GJ, Castro CC, et al. Complex partial seizures and mesial temporal sclerosis, evaluation with Fast Spin Echo magnetic resonance imaging. Radiology 1993; 189: 835-42.

15. Jack CR Jr, Sharbrough RW, Cascino GD, Hirschorn KA, O'Brien PC, Marsh WR. MRI-based hippocampal volumetry: correlation with outcome after temporal lobectomy. Ann Neurol 1992; 31:138-46.

16. Spencer SS, McCarthy G, Spencer DD. Diagnosis of medial temporal lobe seizure onset: relative specificity and sensitivity of quantitative MRI. Neurology 1993; 43: 2117-2435

17. Cascino GD. Neuroimaging in partial epilepsy: structural MRI. J Epilepsy 1998;11:121-9.

18. Cascino GD, Trenerry MR, So EL, et al. Routine EEG and TLE: relation to long-term EEG monitoring, quantitative MRI, and operative outcome. Epilepsia 1996; 37: 651-6.

19. Radakhrisnan K, So EL, Silbert PL, et al. Predictors of anterior temporal lobectomy for intractable epilepsy: a multivariate study. Neurology 1998; 51: 465-71.

20. Cascino GD, Jack CR, Parisi JE, et al. MRI-based hippocampal volumetric studies in TLE: pathological correlation. Ann Neurol 1991;30:31-36

21. Cendes F, Arruda F, Dubeau F, Gotman J, Andermann F, Arnold D. Relationship between mesial temporal atrophy and ictal and interictal EEG findings: results of 250 patients (abstract). Epilepsia 1995; 36: (Supp 14): 23.

22. Cambier DM, Cascino GD, So EL, Marsh WR. Video-EEG monitoring in patients with hippocampal atrophy. Acta Neurol Scand 2001; 103: 231-7.

23. Muttaqin Z. Surgery for temporal lobe epilepsy in Semarang, Indonesia: The first 56 patients with follow up longer than 12 months. Neurology Asia 2006; 11:31-36

24. Wieser HG, Silfvenius H. Overview: epilepsy surgery in developing countries. Epilepsia 2000; 41(Suppl4): S3-S9.

25. Sanyal SK. Relieving the burden of intractable epilepsy in India and other developing countries: the case for two tier epilepsy centers. Neurology Asia 2007; 12 (Supplement 2): 23-28

26. Selladurai BM. Epilepsy Surgery service in Malaysia. Neurology Asia 2007; 12: (Supplement 2):39-41

27. Engel J Jr, van Ness P, Rasmussen TB, et al. Outcome with respect to epileptic seizures. In: Engel J Jr, ed:Surgical treatment of epilepsies. 2nd Ed. New York: Raven Press, 1993: 609-21.

28. Wyler AR. Surgical Treatment on temporal lobe epilepsy. Neurosurg Quarterly 1992; 4: 214-32.

29. Gloor P, Olivier A, Ives J. Prolonged seizure monitoring with stereotactically implanted depth electrodes in patients with bilateral interictal temporal epileptic foci: how bilateral is bitemporal epilepsy? Adv Epileptology 1980;10: 83-8.

30. So N, Gloor P, Quesney LF, Jones Gotman M, Olivier A, Andermann F. Depth electrode investigations in patients with bitemporal epileptiform abnormalities. Ann Neurol 1989; 25: 423-31.

31. So N, Olivier A, Andermann F Gloor P, Quesney LF. Results of surgical treatment in patients with bitemporal epileptiform abnormalities. Ann Neurol 1989; 25: 432-9.

32. Holmes MD, Dodrill CB, Ojemann LM, Ojemann GA. Five year outcome after epilepsy surgery in non-monitored and monitored surgical candidates. Epilepsia 1996; 37: 748-52.
33. Velloglu SK, Ozmenoglu M, Komsuoglu SS. EEG investigation of TLE. Clin Electroencephalogr 1997; 28: 121-6.

34. Die WB, Najen I, Mohammed A, et al. Interictal EEG, hippocampal atrophy, and cell densities in Hippocampal sclerosis associated with microscopic cortical dysplasia. J Clin Neurophysiol 2002; 19: 157-62.

35. Gilliam F, Bowling S, Bilir E, et al. Association of combined MRI, interictal EEG, and ictal EEG results with outcome and pathology after temporal lobectomy. Epilepsia 1997; 38: 131520.

36. Sperling MR, O'Connor MJ. Comparison of depth and subdural electrodes in recording temporal lobe seizures. Neurology 1989;39:1290-301.

37. Sakai Y, Nagano H, Sakata A, et al. Localization of epileptogenic zone in TLE by ictal scalp EEG. Seizure 2002; 11: 163-8.

38. Murro AM, Park YD, King DW, et al. Use of scalp-sphenoidal EEG for seizure localization in TLE. J Clin Neurophysiol 1994; 11:216-9.

39. Wieser HG, Siegel AM, Yasargyl GM. The Zurich amygdalohippocampectomy series: A short up-date. Acta Neurochir 1990; 50 (Suppl): 122-7.

40. Hirsch LJ, Spencer SS, Spencer DD, et al. Criteria for and Results of Temporal Lobectomy in patients with Independent bilateral temporal lobe seizure onsets on depth EEG. Epilepsia 1989; 30: 703.

41. Chinvarun Y, Sakulnamaka S. Temporal lobe epilepsy surgery with limited resources: Outcome and economic considerations. Neurology Asia 2004; 9 (Supp 1): 126

42. Srikijvilaikul T, Bunyarattavej K, Deesudchit T, et al. Outcome after temporal lobectomy for hippocampal sclerosis: Chulalongkorn Comprehensive Epilepsy Program experiences. Neurology Asia 2004; 9 (Supp 1): 127

43. Panda S, Radhakrishnan K, Sarma PS. Mortality in surgically versus medically treated patients with medically refractory temporal lobe epilepsy. Neurology Asia

44. Cramer JA. Quality of life for people with epilepsy. Neurologic Clinics 1994; 12(1):1-13

45. Sillanpaa M, Jalava M, Kaleva O, Shinnar S. Longterm prognosis of seizures with onset in childhood. N Eng J Med 1998; 338: 1715-22.

46. Kwan P, Brodie MJ. Early identification of refractory epilepsy. N Engl J Med 2000;342: 314-9.

47. Radhakrishnan K, Rao MB. Is epilepsy surgery possible in countries with limited resources? Epilepsia 2000; 41(Suppl 4):S31-4

48. Tasch E, Cendes F, Li LM, Dubeau F, Andermann F, Arnold DL. Neuroimaging evidence of progressive neuronal loss and dysfunction in TLE. Ann Neurol 1999; 45: 568-76.

49. Semah F, Picot M-C, Adam C, et al. Is the underlying cause of epilepsy a major prognostic factor for recurrence? Neurology 1998; 51:1256-62.

50. Perucca A, Beghi E, Dulac O, Shorvon S, Tomson T. Assessing risk and benefit ratio in antiepileptic drug therapy. Epilepsy Res 2000; 41: 107-39.

51. Muttaqin Z. Neuroimaging in epilepsy: MRI evaluation in complex partial seizures. Neurology Asia 2007; 12 (Supplement 1): 97

52. Kinirons P, McCarthy M, Doherty CP, Delanty N. Predicting drug-resistant patients who respond to add-on therapy with levetiracetam. Seizure 2006 Jun 9; [Epub ahead of print].

53. Trevathan E, Gilliam F. Lost Years: delayed referral for surgically treatable epilepsy. Neurology 2003; 61: 432-3.

54. Yoon HH, Kwon HL, Mattson RH, Spencer DD, Spencer SS. Long term seizure outcome in patients initially seizure-free after resective epilepsy surgery. Neurology 2003; 61:445-50. 